\title{
Energy Storage System Based on Cascaded Multilevel Inverter with
}

\section{Decoupled Energy Balancing Control}

\author{
Yuanzhi Cao ${ }^{1}$, Junfen Lu ${ }^{1}$, Jun Zhuang ${ }^{1}$, YueLong Li ${ }^{1}$, Wei Jiang ${ }^{2}$, Hui Zhao ${ }^{1}$ \\ ${ }^{1}$ China Electric Power Research Institute, Nanjing 210003, China \\ ${ }^{2}$ Department of Electrical Engineering, Southeast University, Nanjing 210096, China
}

\begin{abstract}
This paper presents a three phase cascaded multilevel inverter based supercapacitor (SC) energy storage system with novel structure and control strategy to maintain the energy balance of between phases. Every two phases are coupled with a series LC filter. With the filter, SC cells in different phases could exchange energy with an auxiliary power flow at high frequency. The auxiliary power flow is orthogonal to the primary power flow. The phase difference between high frequency voltage and current components of each phase determines whether the energy is absorbed into or released from its SC cells. Unlike traditional energy balancing strategies, the proposed method is independent to the fundamental real power drawn by the energy storage system. Simulation results confirmed the effects of proposed theories.
\end{abstract}

\section{Introduction}

Cascaded multilevel inverters (CMI) are popular in high-power applications such as static synchronous compensation (STATCOM), hybrid renewable energy system, and motor drive system [1]-[4]. The application of CMI in energy storage system has merits like reduced switching frequencies without increasing harmonic filtering requirements, inherent redundancy, mediumvoltage adaption without transformers and cost reduction due to using low-cost-semiconductor [4]. A typical CMI based three phase energy storage system is shown in Figure 1. Every standard module in the system is consisted of an energy storage component and an $\mathrm{H}-$ bridge inverter.

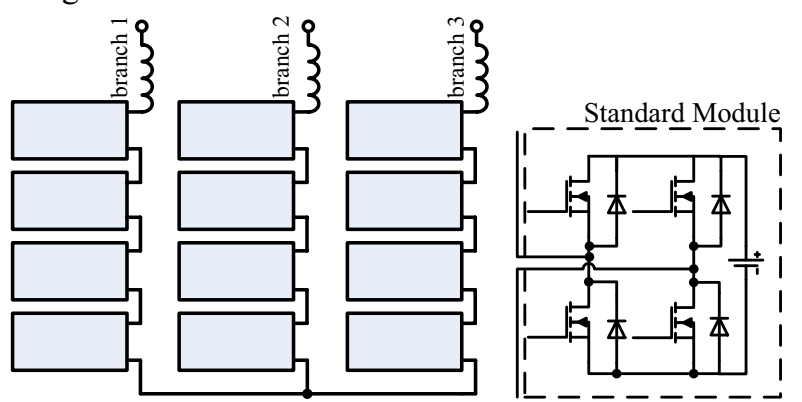

Figure 1. Cascaded multilevel inverter based three phase energy storage system

However, the energy stored in every energy storage component, i.e. supercapacitor or battery cells are unbalanced because of manufacturing variance, difference of converter loss and fault [5]. The traditional voltage balancing approaches of CMI make small alternations on the switching signals [6]. The power and speed of the energy balancing process depends on the delivered active/reactive power of the inverter. When there is no power transferred or the inverter is working in islanded mode, the energy balancing methods will properly fail [7].
This paper presents a novel energy balancing strategy based on the principle of orthogonal power flow. By introducing three high frequency auxiliary power flows into the three-phase cascaded multilevel inverter based SC energy storage system, the energy balancing control could be decoupled from the active/reactive power flow of fundamental frequency.

\section{Topology of the novel energy storage system}

If the voltage/current is consisted of sinusoidal functions at different frequencies, the active power resulted from the non-sinusoidal voltage and current can be defined as the mean value of the production of the instantaneous value of voltage and current [8].

$$
\begin{gathered}
v(t)=\sum_{n=1}^{N} \sqrt{2} V_{n} \cos \left(\omega_{n} t+\theta_{n}\right) \\
i(t)=\sum_{n=1}^{N} \sqrt{2} I_{n} \cos \left(\omega_{n} t+\varphi_{n}\right) \\
P=\sum_{n=1}^{N} V_{n} I_{n} \cos \left(\theta_{n}-\varphi_{n}\right)
\end{gathered}
$$

According to the orthogonality of trigonometric function, the power produced by different frequency components is orthogonal to each other. So the inverter in every branch of the three phase energy storage system can generate active power at one frequency to supply loads while absorbing power at another frequency if there are proper route. Based on this theory, a novel structure of three phase SC energy storage system with star configuration in islanded mode is proposed in Figure 2.

\footnotetext{
a Corresponding author: author@e-mail.org
}

This is an Open Access article distributed under the terms of the Creative Commons Attribution License 4.0, which permits unrestricted use, distribution, and reproduction in any medium, provided the original work is properly cited. 


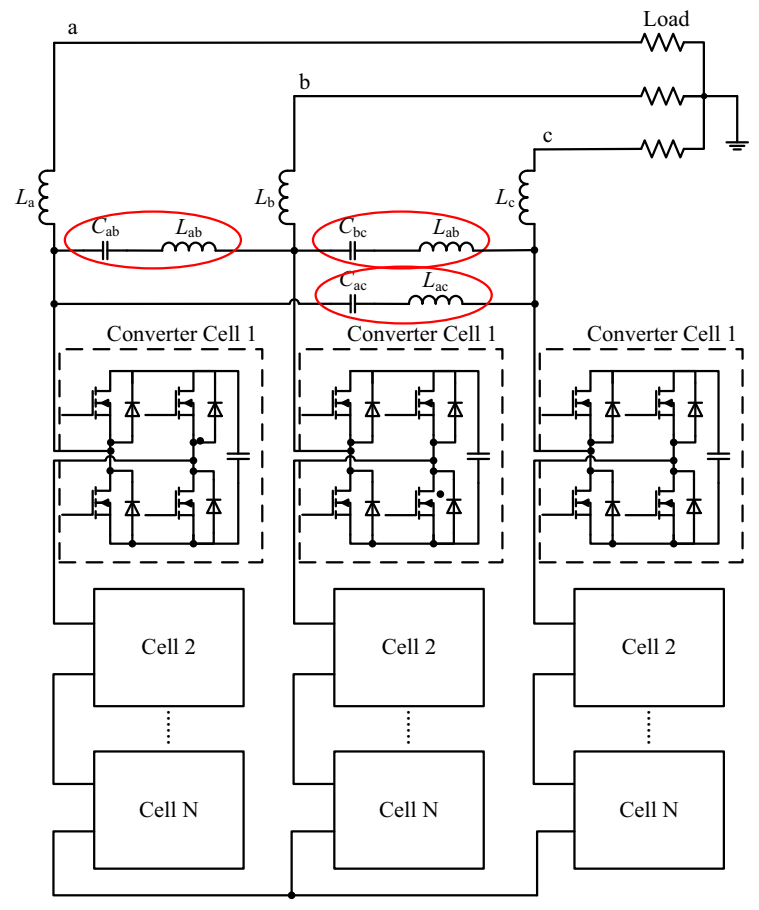

Figure 2. The topology of proposed SC energy storage system is islanded mode

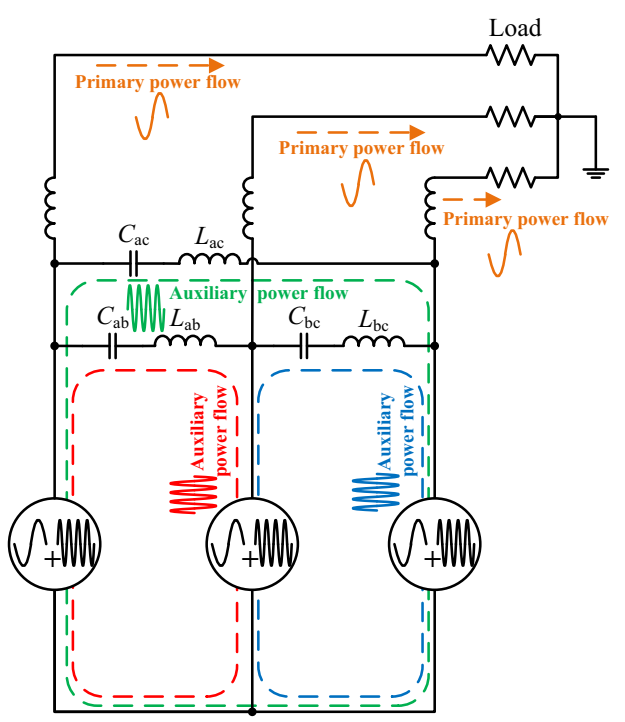

Figure 3. Simple model of primary and auxiliary power flow

A series LC filter is placed between every two branches. Every LC filter provides an auxiliary power loop. The duty cycle of the PWM generator of each converter cell is modulated with a non-sinusoidal signal added up with two sinusoidal waves of different frequencies. The first frequency is the primary frequency which would be the line frequency $(50 \mathrm{~Hz}$ or $60 \mathrm{~Hz})$. The second frequency is the auxiliary frequency which would be set to harmonics/subharmonics of the line voltage. Since the LC filter is placed between the series connected converter cells and the filtering inductor connected to the AC line, the power flow at the auxiliary frequency will not leak to the AC line. The simple model of the primary and auxiliary power flows is shown in Figure 3.

\section{Control strategy design}

\subsection{Voltage Balancing Control}

The SC output power of branch $n(n=1,2,3) P_{\mathrm{n}}$ is consisted of two components. The primary component $P$ prim $n$ is determined by the AC load and it is positive (SC discharge). Since the terminal voltage of SC represents its energy. The auxiliary component $P_{\text {aux } \_ \text {n }}$ is determined by the SC voltage error between different branches.

$$
\begin{gathered}
P_{n}=P_{p r i m \_n}+P_{a u x \_n} \\
P_{\text {prim } \_n}=V_{\text {prim } \_n} I_{\text {prim } \_n} \cos \left(\theta_{\text {prim } \_n}-\varphi_{\text {prim } \_n}\right) \\
P_{\text {aux } \_n}=V_{\text {aux } \_n} I_{\text {aux } \_n} \cos \left(\theta_{\text {aux } \_n}-\varphi_{a u x \_n}\right)
\end{gathered}
$$

When the overall SC cells voltage $v_{\text {sc } n}$ of branch $n$ is below average voltage $v_{\mathrm{sc} \text { ave }}$ of three branches, $P_{\mathrm{n}}$ should be negative so $\mathrm{SC}$ cells are charged by the auxiliary power flow. When $v_{\mathrm{sc} \_n}$ is above $v_{\text {sc_ave }}, P_{\mathrm{n}}$ should be positive so SC cells discharge power to the auxiliary power flow. The polarity of $P{ }_{\mathrm{n}}$ is determined by the magnitude and polarity of $P_{\text {aux_n }}$. Equation (6) implies that when $\theta_{\text {aux } \_n}-\varphi_{\text {aux } \_n}=0, \quad P$ aux $\_$n $_{\text {is }}$ is forward maximum; when $\theta_{a u x_{\_} n}-\varphi_{\text {aux } \_n}=\pi, P_{\text {aux } n \text { }}$ is reverse maximum; when $\theta_{a u x_{-} n}-\varphi_{a u x_{n}}=\frac{\pi}{2}, P_{\text {aux_n }}$ is zero. So by adjusting the magnitude of $v_{a u x_{-} n}(t)$ and the shifted phase between $i_{\text {aux } \_n}(t)$ and $v_{\text {aux } \_n}(t), P_{\text {aux } \_ \text {n }}$ can be controlled. The diagram of the voltage balancing controller is shown in Figure 4.

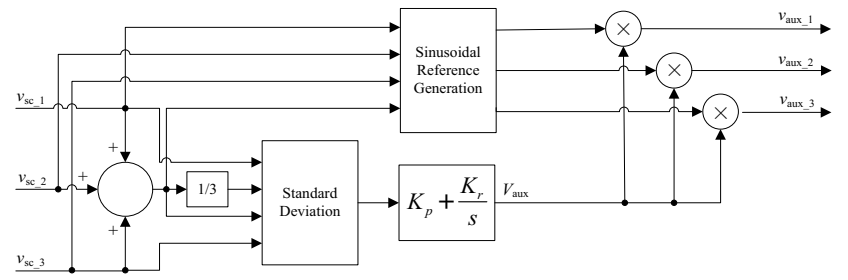

Figure 4. Diagram of voltage balancing controller

$$
\sqrt{\frac{\left(v_{s c_{\_} 1}-v_{s c_{\_} a v e}\right)^{2}+\left(v_{s c_{-} 2}-v_{s c_{-} a v e}\right)^{2}+\left(v_{s c_{-} 3}-v_{s c_{\_} a v e}\right)^{2}}{3}}
$$

The standard deviation of the SC voltage of the three branches in equation (7) and a PI controller determines the magnitude $V_{\text {aux }}$ of the three sinusoidal auxiliary voltage components $v_{\text {aux } 1} \sim v_{\text {aux } 3}$. A table of relationship between auxiliary power flow and phase differences of three branches based on equations (8) (13) is stored in the sinusoidal reference generation module. The module generates three sinusoidal signals at auxiliary frequency with phase differences between $\theta_{1}, \theta_{2}, \theta_{3}$. The auxiliary frequency of the signals needs to be actively controlled to track the resonant frequency of the series LC filter. Thus the impedance to the auxiliary power flow is minimum. 


$$
\begin{gathered}
v_{a u x_{\_} 1}(t)=V_{\text {aux }} \sin \left(\omega t+\theta_{1}\right) \\
v_{a u x_{\_} 2}(t)=V_{\text {aux }} \sin \left(\omega t+\theta_{2}\right) \\
v_{a u x_{\_} 3}(t)=V_{\text {aux }} \sin \left(\omega t+\theta_{3}\right) \\
P_{a u x_{\_} 1}(t)=-\frac{V_{a u x}^{2}}{R} \cos \frac{2 \theta_{1}-\theta_{2}-\theta_{3}}{2} \cos \frac{\theta_{3}-\theta_{2}}{2} \\
P_{a u x_{\_} 2}(t)=-\frac{V_{a u x}^{2}}{R} \cos \frac{2 \theta_{2}-\theta_{1}-\theta_{3}}{2} \cos \frac{\theta_{3}-\theta_{1}}{2} \\
P_{a u x_{\_} 3}(t)=-\frac{V_{a u x}^{2}}{R} \cos \frac{2 \theta_{3}-\theta_{1}-\theta_{2}}{2} \cos \frac{\theta_{2}-\theta_{1}}{2}
\end{gathered}
$$

\subsection{Primary Power Flow Control}

The primary power flow is designed to provide a stable $\mathrm{AC}$ voltage at line frequency to $\mathrm{AC}$ loads. Since the primary power flow and auxiliary power flow are orthogonal to each other. The primary power flow controller has traditional double loop structure as shown in Figure 5. The error between output voltage $v_{\mathrm{c}}$ and is reference $v^{*}$ is compensated by a quasi-PR controller. The output of quasi-PR controller is send to PI controller of the current loop. The output of the current loop is added with $v_{\text {aux }}$ from the voltage balancing controller and become the modulation wave of the phase shift SPWM module.

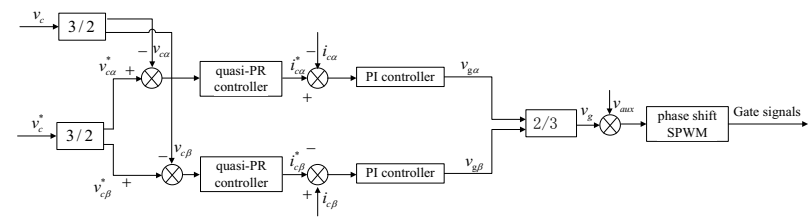

Figure 5. Diagram of primary power controller

\section{Simulation and discussion}

To verify the performance of the proposed energy storage system, a series of simulation are developed. Each branch of the inverter contains three H-bridge inverter. Each $\mathrm{H}$-bridge inverter is paralleled with a $2 \mathrm{~F}$ SC cell as energy source. The primary and auxiliary frequencies are set to $50 \mathrm{~Hz}$ and $1000 \mathrm{~Hz}$. The initial SC voltages of the three branches are set to $900 \mathrm{~V}, 870 \mathrm{~V}$ and $850 \mathrm{~V}$ so their stored energy are unbalanced.

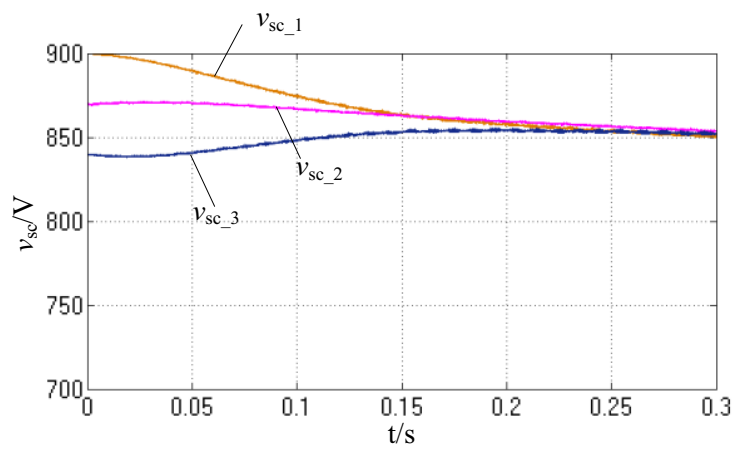

Figure 6. The DC voltage waveform of three branches

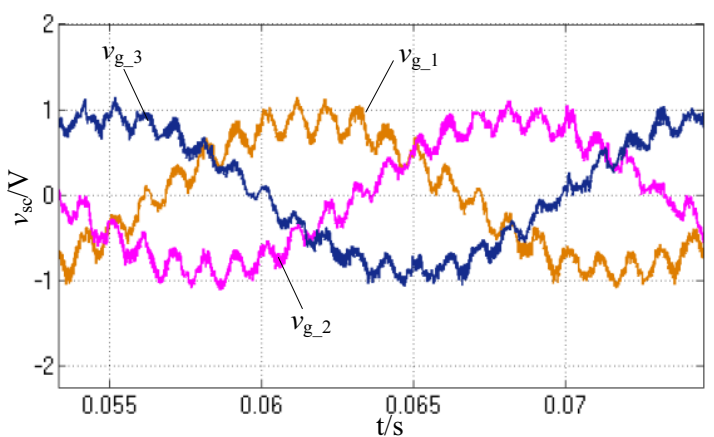

Figure 7. The modulation voltage waveform of three phases
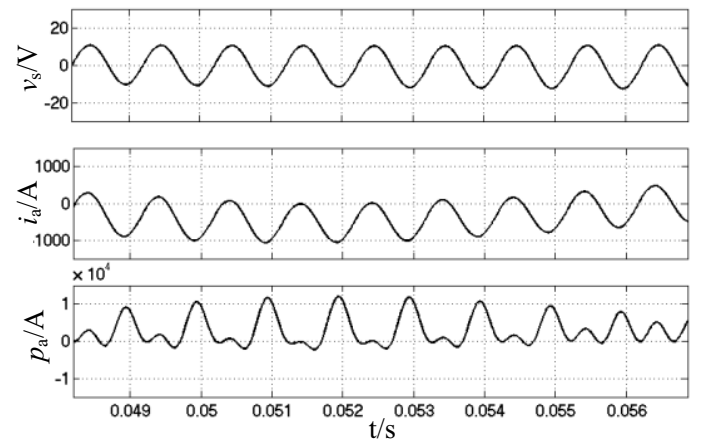

Figure 8. The terminal voltage/current/power waveforms of phase A
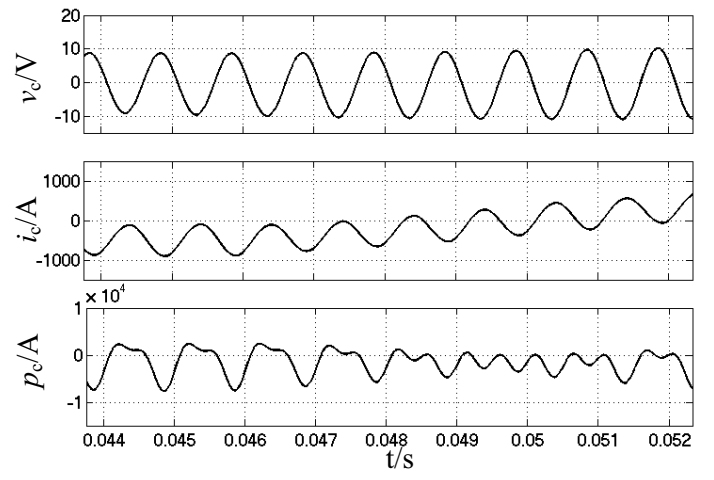

Figure 9. The terminal voltage/current/power waveforms of phase B

Figure 6 shows the overall SC cells voltage waveform of the three branches. The voltages are eventually balanced since energy is exchanged under control within auxiliary power loop. The modulation waveforms of three phases are shown in Figure 7. A high frequency $(1000 \mathrm{~Hz})$ sinusoidal signal is added to the $50 \mathrm{~Hz}$ modulation signal. The phase difference between auxiliary voltage and current at $1000 \mathrm{~Hz}$ of phase $\mathrm{A}$ is 0 as shown in Figure 8 . So the auxiliary power at is positive, implies that $\mathrm{SC}$ cells in phase A generate balancing energy. The phase difference between auxiliary voltage and current of phase $\mathrm{C}$ is $\pi$ as shown in Figure 9. So the auxiliary power is negative, implies that $\mathrm{SC}$ cells in phase $\mathrm{C}$ absorb balancing energy. So the voltage of SC cells in phase $\mathrm{C}$ increase while providing real power to AC load.

\section{Conclusion}

In this paper, a novel three phase energy storage system based on cascaded multilevel inverter is proposed. Series LC filters are places between every two phases so 
auxiliary power flow at high frequency could exchange energy between SC cells in different phase. The topology, orthogonal power flow theory and controller design is introduced. Simulation results verified proposed theories.

\section{References}

[1] J. Rodriguez, J. S. Lai, and F. Z. Peng, "Multilevel inverters: A survey of topologies, controls and applications," IEEE Trans. Ind. Electron., vol. 49, no. 4, pp. 724-738, Aug. 2002.

[2] S. Lu, K. Corzine, and M. Ferdowsi, "High power motor drives based on hybrid multilevel converters and direct torque control," inProc. IEEE APEC, 2007, pp. 1077-1083.

[3] M. D. Manjrekar, P. K. Steimer, and T. A. Lipo, "Hybrid multilevel power conversion system: A competitive solution for high-power applications," IEEE Trans. Ind. Appl., vol. 36, no. 3, pp. 834-841, May/Jun. 2000.

[4] L. Baruschka and A. Mertens, "Comparison of cascaded hbridge converters and modular multilevel converters for the use in medium voltage grid connected battery energy storage systems," in 21 st International Conference on Electricity Distribution CIRED'11, 2011, paper 1098.

[5] F. Z. Peng, "A generalized multilevel inverter topology with self voltage balancing," IEEE Trans. Ind. Appl., vol. 37, no. 2, pp. 611-618, Mar./Apr. 2001.

[6] L. Maharjan, S. Inoue, and H. Akagi, "State-of-Charge (SOC)-balancing control of a battery energy storage system based on a cascaded PWM converter," IEEE Trans. Power Electron., vol. 24, no. 6, pp. 1628-1636, Jun. 2009.

[7] D. Soto, R. Peña and P. Wheeler, "Decoupled Control of Capacitor Voltages in a PWM Cascade StatCom", IEEE Power Electronics Specialists Conference, 2008, pp. 1384 1389.

[8] Jan A. Ferreira, "The Multilevel Modular DC Converter", IEEE Trans. Power Electron., vol. 28, no. 10, pp. 44604465, Oct. 2013. 